\title{
Electric-Field-Induced Enhancement/Quenching of Photoluminescence of $\pi$-Conjugated Polymer S3-PPV: Excitation Energy Dependence
}

\author{
Mohan Singh Mehata, ${ }^{\dagger}$ Chain-Shu Hsu, Yuan-Pern Lee, and Nobuhiro Ohta*, \\ Research Institute for Electronic Science (RIES), Hokkaido University, Sapporo 001-0020, Japan, and \\ Department of Applied Chemistry and Institute of Molecular Science, National Chiao Tung University, \\ Hsinchu 30010, Taiwan
}

Received: December 27, 2009; Revised Manuscript Received: April 5, 2010

\begin{abstract}
The effects of electric field on absorption and photoluminescence (PL) of films of sulfide-substituted PPV derivative S3-PPV, poly[2-(phenyl)-3-(4'-(3,7-dimethyloctyloxy)phenyl)-1,4-phenylenevinylene-co-2-(11'-decyl sulfanylundecanyloxy)-5-methoxy-1,4-phenylene vinylene], were investigated. Electroabsorption (E-A) and electrophotoluminescence (E-PL) responses of S3-PPV show the Stark shifts, indicating a significant alternation in the molecular polarizability $(\Delta \bar{\alpha})$ associated with the optical transitions. Field-induced enhancement or quenching is also observed for PL of S3-PPV, depending on the photoexcitation energy, whereas the shape of the PL spectra is independent of the excitation wavelength. The field effects on the decay profiles of PL indicate that the quenching results from a diminished population of the emitting states on excitation at 300 $\mathrm{nm}$, whereas the PL is enhanced on excitation at $471 \mathrm{~nm}$ because the emitting state has an increased lifetime. The efficiency of field-assisted generation of electron-hole pairs produced through excitons monotonically increases with increasing excitation energy, and the nonradiative decay rate in the emitting state is diminished by electric fields in S3-PPV. The photoirradiation of S3-PPV in ambient air resulted in rapid degradation of the polymer film.
\end{abstract}

\section{Introduction}

Having highly extended $\pi$-conjugation systems in their main chain, PPV, poly ( $p$-phenylene vinylene), and its derivatives are important semiconductor polymers for optical applications. With spin-casting, they are readily fabricated into large active areas in the range of thickness from less than 1 to $\sim 100 \mu \mathrm{m}$ and exhibit a large efficiency for conversion between light and electricity. ${ }^{1}$ These polymers also possess the advantages of having efficient and versatile photoluminescence (PL) and electroluminescence (EL). Being electroluminescent, they have received much attention because of their wide applications in electronic and optoelectronic devices such as light-emitting diodes, thin-film transistors, photovoltaic cells, chemical and biological sensors, nonlinear optical devices, and electrochemical cells. ${ }^{1-3}$ The optical and electronic properties of electronically excited states of least energy are important in both electroluminescent and photovoltaic processes. The degradation of these luminescent conducting polymers is a key factor determining their possible commercial applications. ${ }^{4-6}$ Understanding the mechanism governing the efficiency and durability at a molecular level for polymers under operating conditions might provide important clues for an optimization of the chemical structure. ${ }^{6,7}$

PPV is poorly soluble in most organic solvents. To improve its solubility for practical processing, derivatives of PPV with active side groups were synthesized; thermal, electrochemical, and EL properties in device configurations of synthesized PPV derivatives were characterized. ${ }^{8,9}$ The absorption and fluorescence of PPV and its soluble derivatives MEH-PPV, poly[2-methoxy-5-(2'-ethyl-

\footnotetext{
* To whom correspondence should be addressed. E-mail: nohta@ es.hokudai.ac.jp.

Hokkaido University.

* National Chiao Tung University

\& Permanent address: Photophysics Laboratory, Kumaun University, Nainitial 263002, Uttarakhand, India.
}

\section{CHART 1: Chemical Structure of S3-PPV}

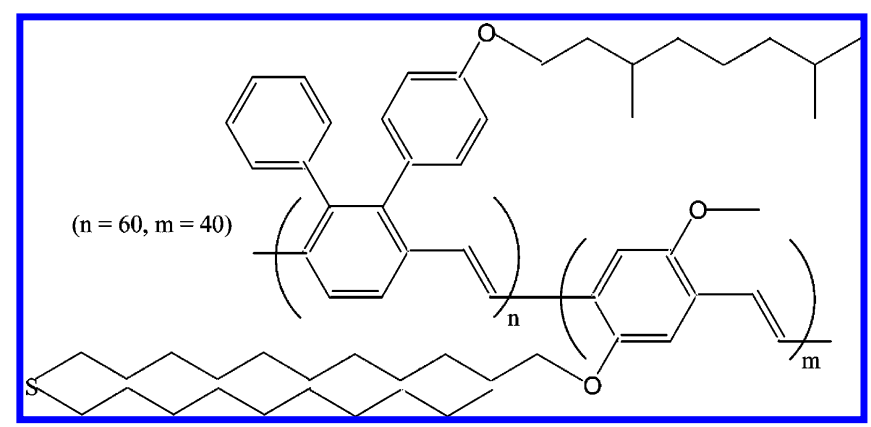

hexyloxy)-p-phenylenevinylene, were investigated in the presence of an electric field; values of the binding energy and the variation of the dipole moment and polarizability between the emitting and the ground states were estimated. ${ }^{10-13}$

One PPV derivative, S3-PPV, having sulfide long chains is soluble in common organic solvents; the chemical structure is shown in Chart 1. Using S3-PPV in double-layer light-emitting devices, a maximum luminescence of $6073 \mathrm{~cd} \mathrm{~m}^{-2}$ with a current yield of $0.82 \mathrm{~cd} \mathrm{~A}^{-1}$ was obtained. ${ }^{8}$ In relation to the stability and reliability of the EL devices, the effects of an electric field on nonradiative processes and emission intensity are important because intense electric fields are invariably applied during the operation of EL devices. In this work, we have examined the effects of an external electric field on the absorption and photoluminescence spectra and on the decay profile of photoluminescence (PL) of S3-PPV films under vacuum conditions. Electric-field-induced quenching or enhancement of PL intensity of S3-PPV was observed, depending on the wavelength of the excitation light. Observed decay profiles show that, upon irradiation at $300 \mathrm{~nm}$, the field-induced quenching arises from a decreased initial population, whereas 
upon irradiation at $471 \mathrm{~nm}$, the enhancement results from a fieldinduced increase of the PL lifetime. We also examined the stability of PL upon photoirradiation under ambient air conditions.

\section{Experimental Section}

S3-PPV was synthesized with a procedure described in Supporting Information. A sample film of S3-PPV was deposited on an indium-tin oxide (ITO)-coated silica substrate by spincoating from toluene solution; a polymer film of PMMA, poly(methyl methacrylate), was then deposited on the S3-PPV film as an insulator film. From the UV-visible absorption intensity, the thickness of the S3-PPV film was determined to be $20-50 \mathrm{~nm}$. The thickness of the insulating PMMA film, measured with an interferometric microscope (Nano Spec/AFT010-0180, Nanometric), was typically $0.5 \mu \mathrm{m}$. A semitransparent aluminum film (Al) was further deposited on the PMMA film with vacuum vapor deposition. ITO and Al served as electrodes. The sample preparation and experimental procedures for measurements of electroabsorption (E-A) and electrophotoluminescence (E-PL) spectra, that is, plots of the field-induced change in absorption intensity and PL intensity, respectively, as a function of wavenumber, are reported in detail elsewhere..$^{7,14}$ E-PL, E-A, and electrophotoluminescence excitation responses were obtained at the second harmonic of the modulation frequency of the applied electric field, typically at $40 \mathrm{~Hz}$. Hereafter, $F$ denotes applied electric field, and its strength is represented by the rms value.

To measure the profile of photoluminescence decay, we used the second or the third harmonic of a mode-locked Ti:sapphire laser (Spectra Physics, Tsunami, pulse width $\sim 200 \mathrm{fs}$ ) as an excitation source. Fluorescence from the sample was detected with a microchannel plate photomultiplier (Hamamatsu, R3809U52) and fed to a time-to-amplitude converter of a single-photoncounting system. Fluorescence decays were obtained with a multichannel pulse-height analyzer (MCA). To measure the effect of electric field on the decay profile, we applied a modulated voltage to the sample, ${ }^{15}$ this voltage was a rectangular wave of positive, zero, negative, and zero bias in sequence. The duration of each segment was $30 \mathrm{~ms}$, of which the first $3 \mathrm{~ms}$ was a dead time to avoid an overshooting effect immediately after the alteration of the applied voltage. The memory channel of the MCA was divided into four segments. The switching of the MCA memory channel was synchronized with the modulated applied voltage. Four decays were collected, corresponding to positive, zero, negative, and zero sample biases, respectively. These decays were stored in separate memory segments of the MCA.

\section{Results and Discussion}

A. Effect of Electric Field on Absorption Spectra. Figure 1shows absorption and polarized E-A spectra of an S3-PPV film in a range of $16000-34000 \mathrm{~cm}^{-1}$ obtained with a field strength of $0.7 \mathrm{MV} \mathrm{cm} \mathrm{cm}^{-1}$. The spectrum shows an intense broad absorption with a maximum at $22270 \mathrm{~cm}^{-1}(449 \mathrm{~nm})$. This band is reproduced by a superposition of two Gaussian bands, $\mathrm{G}_{1}$ and $\mathrm{G}_{2}$, which might correspond to two different transitions. Two additional Gaussian bands, $\mathrm{G}_{3}$ and $\mathrm{G}_{4}$, are required to reproduce the complete absorption spectrum (Figure 1a).

Polarized E-A spectra were recorded at the normal angle of incidence to the surface $\left(\chi=90^{\circ}\right)$ and at the magic angle $\left(\chi=54.7^{\circ}\right) ; \chi$ is the angle between the direction of the applied electric field and the electric vector of the incident light. The E-A spectra recorded at both angles are similar, indicating a

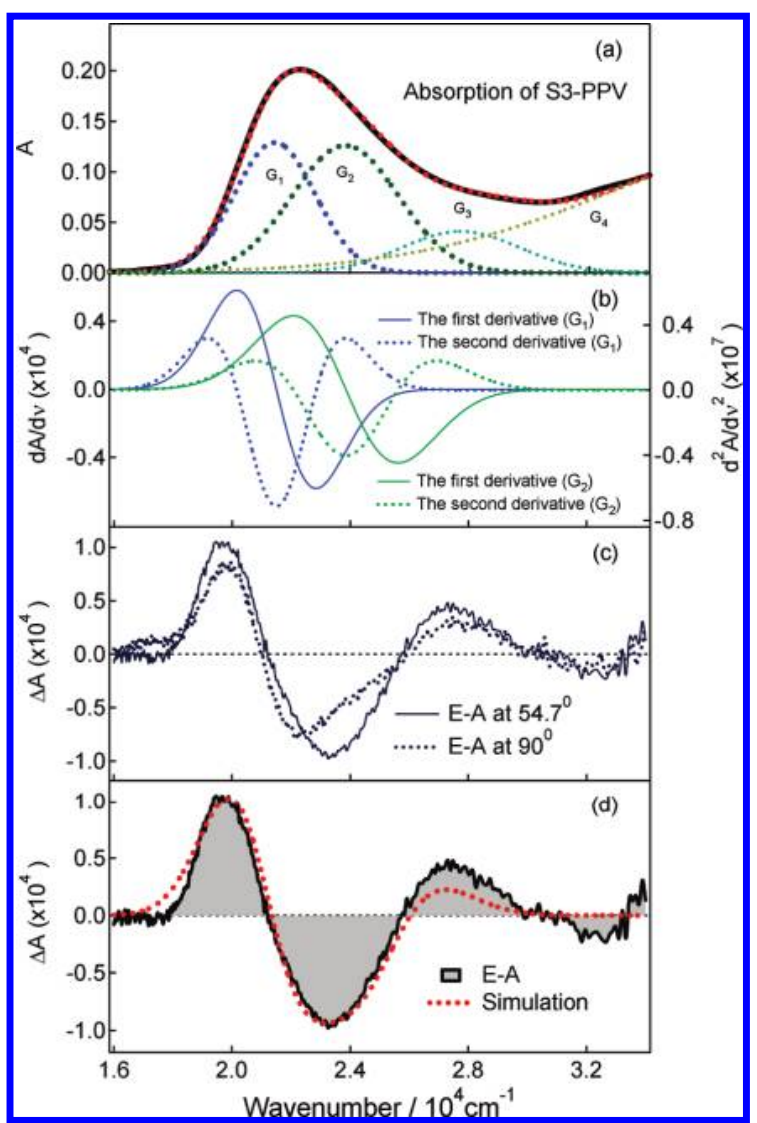

Figure 1. (a) Absorption spectrum of an S3-PPV film, (b) derivative spectra of the $\mathrm{G}_{1}$ and $\mathrm{G}_{2}$ bands, (c) electroabsorption (E-A) spectra observed at angles $\chi=90$ and $54.7^{\circ}$ with a field strength of $0.7 \mathrm{MV}$ $\mathrm{cm}^{-1}$, and (d) simulated E-A spectrum (dotted line) with the spectrum observed with $\chi=54.7^{\circ}$ (shaded line). A dotted red line in (a) shows the spectrum simulated with a sum of $\mathrm{G}_{1}-\mathrm{G}_{4}$ bands; these bands are indicated with dotted lines.

nearly isotropic distribution of the S3-PPV sample in both the absence and the presence of an external field.

The field-induced change in absorption intensity (E-A) or photoluminescence intensity (E-PL) at frequency $v$, observed at the second harmonic of the modulation frequency of the applied electric field, is given by the following equations ${ }^{16-18}$

$$
\Delta A(v)=(f F)^{2}\left[A_{\chi} A(v)+B_{\chi} v \frac{d}{d v}\left\{\frac{A(v)}{v}\right\}+C_{\chi} v \frac{d^{2}}{d v^{2}}\left\{\frac{A(v)}{v}\right\}\right]
$$

$$
\begin{aligned}
\Delta I_{\mathrm{PL}}(v)=(f F)^{2}\left[A_{\chi}^{\prime} I_{\mathrm{PL}}(v)+B_{\chi}^{\prime} v^{3} \frac{d}{d v}\left\{\frac{I_{\mathrm{PL}}(v)}{v^{3}}\right\}+\right. \\
\left.C_{\chi}^{\prime} v^{3} \frac{d^{2}}{d v^{2}}\left\{\frac{I_{\mathrm{PL}}(v)}{v^{3}}\right\}\right]
\end{aligned}
$$

in which $f$ is the internal field factor and $F$ is the electric field strength. Coefficients $A_{\chi}$ and $A_{\chi}{ }^{\prime}$ correspond to the field-induced change in the absorption and PL intensity, respectively, whereas coefficients $B_{\chi}\left(B_{\chi}{ }^{\prime}\right)$ and $C_{\chi}\left(C_{\chi}{ }^{\prime}\right)$ correspond to the spectral shift and spectral broadening of the E-A/E-PL spectra resulting from the difference in molecular polarizability $(\Delta \alpha)$ and in electric dipole moment $(\Delta \mu)$, respectively, following the optical transition. These coefficients are expressed as 


$$
\begin{gathered}
B_{\chi}=\frac{\Delta \bar{\alpha}}{2 h c}+\left\{\frac{\left(\Delta \alpha_{m}-\Delta \bar{\alpha}\right)\left(3 \cos ^{2} \chi-1\right)}{10 h c}\right\} \\
C_{\chi}=|\Delta \mu|^{2}\left\{\frac{5+\left(3 \cos ^{2} \chi-1\right)\left(3 \cos ^{2} \eta-1\right)}{30 h^{2} c^{2}}\right\}
\end{gathered}
$$

in which $|\Delta \mu|=\left|\mu_{\mathrm{e}}-\mu_{\mathrm{g}}\right|$, and $\Delta \bar{\alpha}=1 / 3 \operatorname{Tr}(\Delta \alpha)=\alpha_{\mathrm{e}}-\alpha_{\mathrm{g}}$, where subscripts e and $\mathrm{g}$ represent the excited and ground states, respectively; $\Delta \alpha_{m}$ represents the diagonal component of $\Delta \bar{\alpha}$ with respect to the direction of the transition dipole moment; $\eta$ is the angle between the direction of $\Delta \mu$ and the transition dipole moment.

In the region of $16000-25000 \mathrm{~cm}^{-1}$, the E-A spectrum follows closely the first derivative of the absorption spectrum. The E-A spectrum is reproduced as a linear combination of the first and second derivative spectra of Gaussian bands $G_{1}$ and $\mathrm{G}_{2}$ shown in Figure 1b. From the fit, the changes in the molecular polarizability $(\Delta \bar{\alpha})$ and dipole moment $(\Delta \mu)$ associated with the optical transition from the ground to the excited state were evaluated for the $G_{1}$ and $G_{2}$ bands, respectively. The results are shown in Table 1 . The $\mathrm{G}_{3}$ and $\mathrm{G}_{4}$ bands were excluded from the simulation. In the region of 25 500-30 000 $\mathrm{cm}^{-1}$, the match between the simulated and observed spectra was less satisfactory, perhaps because bands $\mathrm{G}_{3}$ and $\mathrm{G}_{4}$ were excluded from the fitting or because forbidden transitions became weakly allowed in the presence of an electric field. ${ }^{10}$ Without separation of the absorption spectrum into different bands, the observed E-A spectra could not be reproduced by simulation, as shown in Supporting Information (see Figure S1).

The magnitude of $\Delta \mu$ obtained in the present study is similar to the values obtained from the electroabsorption spectra of the transition between the ground state $\left(\mathrm{S}_{0}\right)$ and the Franck-Condon ${ }^{1} \mathrm{~B}_{\mathrm{u}}$ excited state of derivatives of PPV such as poly[2methoxy,5-(2'-ethylhexoxy)-1,4-phenylene vinylene (MEHPPV) or oligophenylenevinylenes (OPPVs) dissolved in a glassy solvent. ${ }^{12}$ In contrast with $\Delta \mu$, the present value of $\Delta \alpha$ is $0.01-0.1$ times those obtained for the transition between $S_{0}$ and the ${ }^{1} \mathrm{~B}_{\mathrm{u}}$ state of MEH-PPV or OPPVs in a glassy solvent, ${ }^{12,13}$ indicating that the excited state of S3-PPV that shows strong absorption with a maximum $\sim 22500 \mathrm{~cm}^{-1}$ differs somewhat from those of MEH-PPV or OPPVs in a glassy solvent. It is worth mentioning that $\Delta \alpha$ of a polymer film of PPV, $\sim 3300$ $\AA^{3} / f^{2}$, is $10-100$ times as large as the present value, whereas the magnitude of $\Delta \alpha$ of a polymer film of MEH-PPV, $\sim 116$ $\AA^{3} / f^{2}$, is similar to the present value of S3-PPV. ${ }^{19}$ Some difference might reflect the degree of order within the polymer films, though disorder alone fails to account for all differences between PPV and MEH-PPV. ${ }^{19}$

TABLE 1: Magnitudes of $\Delta \bar{\alpha}$ and $\Delta \mu$ of Polymers Obtained from Electroabsorption (E-A) and

\begin{tabular}{|c|c|c|c|c|c|}
\hline \multirow[b]{2}{*}{ polymer } & \multicolumn{2}{|c|}{ E-A } & \multicolumn{2}{|c|}{ E-PL } & \multirow[b]{2}{*}{ ref } \\
\hline & $\Delta \bar{\alpha}\left(\AA^{3} / f^{2}\right)$ & $\Delta \mu(\mathrm{D} / f)$ & $\Delta \bar{\alpha}\left(\AA^{3} / f^{2}\right)$ & $\Delta \mu(\mathrm{D} / f)$ & \\
\hline S3-PPV & $\begin{array}{l}\left(G_{1}\right) 80 \pm 4 \\
\left(G_{2}\right) 29 \pm 2\end{array}$ & $\begin{array}{l}5.9 \pm 0.2 \\
8.8 \pm 0.2\end{array}$ & $840 \pm 10$ & & $\begin{array}{l}\text { this work } \\
\text { this work }\end{array}$ \\
\hline PPV & $\sim 3300$ & & & & $a$ \\
\hline MEH-PPV & $\sim 116$ & & & & $a$ \\
\hline OPPV-3 & $\begin{array}{l}3000 \pm 600 \\
140 \pm 40\end{array}$ & $\begin{array}{c}10 \pm 1 \\
2.0 \pm 0.8\end{array}$ & $2000 \pm 210$ & $14 \pm 3$ & $\begin{array}{l}b, c \\
b\end{array}$ \\
\hline OPPV-5 & $500 \pm 90$ & $4.0 \pm 0.1$ & $500 \pm 60$ & $5.0 \pm 0.1$ & $b$ \\
\hline OPPV-13 & $2000 \pm 200$ & $9.0 \pm 0.1$ & $2900 \pm 850$ & $9.0 \pm 0.2$ & $b$ \\
\hline
\end{tabular}
Electrophotoluminescence (E-PL) Spectra

${ }^{a}$ From ref $19 .{ }^{b}$ From ref $12 .{ }^{c}$ From ref 13.

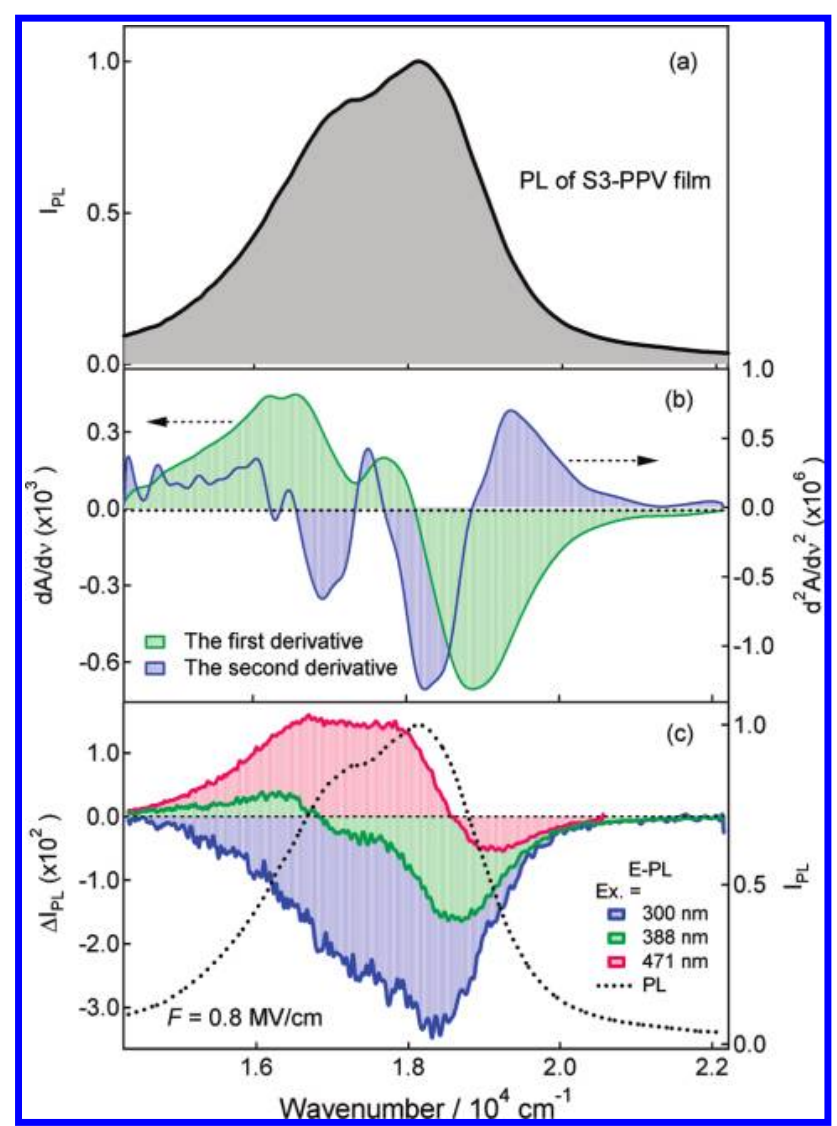

Figure 2. (a) Photoluminescence (PL) spectrum, (b) its first and second derivatives, and (c) electrophotoluminescence (E-PL) spectra of an S3PPV film observed under vacuum conditions with excitation at 300 $\mathrm{nm}$ (shaded blue line), $388 \mathrm{~nm}$ (shaded green line), and $471 \mathrm{~nm}$ (shaded red line) with a field strength of $0.8 \mathrm{MV} \mathrm{cm}^{-1}$.

B. Effect of Electric Field on Spectra and Decay Profile of Photoluminescence (PL). Figure 2 shows the PL spectrum, its derivatives, and E-PL spectra of an S3-PPV film near 298 $\mathrm{K}$ under vacuum condition excited at three different wavelengths with a field strength of $0.8 \mathrm{MV} \mathrm{cm}^{-1}$. In the figure, $\Delta I_{\mathrm{PL}}$ and $I_{\mathrm{PL}}$ represent the field-induced change of PL intensity and the PL intensity at zero field, respectively. E-PL and PL spectra were recorded simultaneously.

For the measurements of the E-PL spectra, excitation wavelengths of 300,388, and $471 \mathrm{~nm}$ were selected, at which the field-induced changes of absorption intensity were negligibly small (Figure 1c). The PL spectra recorded with excitation at these wavelengths have similar shapes with a maximum near $551 \mathrm{~nm}$ and a shoulder near $590 \mathrm{~nm}$ (see Figure 2a), which correspond to the vibronic structure resulting from the transition to various vibrational states of $\mathrm{S}_{0}{ }^{20}$

In contrast with the PL spectra, the E-PL spectra obtained with excitation at 300, 388, and $471 \mathrm{~nm}$ are distinct (Figure 2c). With excitation at $300 \mathrm{~nm}$, the E-PL spectrum is nearly identical to the negative of the PL spectrum. The total integrated intensity of the E-PL spectrum is negative, indicating that the quantum yield of the emission is decreased by application of electric fields. This behavior was observed even when the applied field was as small as $0.4 \mathrm{MV} \mathrm{cm}^{-1}$. The $\Delta I_{\mathrm{PL}}$ integrated over the spectral region $14400-20500 \mathrm{~cm}^{-1}$ is proportional to the square of the applied electric field, which follows the same trend as that reported for polyfluorene. ${ }^{7}$ The fractional field-induced quenching, determined by the ratio of integrated $\Delta I_{\mathrm{PL}}$ to $I_{\mathrm{PL}}$, is $2.7 \pm 0.5 \%$ at a field strength of $0.8 \mathrm{MV} \mathrm{cm}^{-1}$. With excitation at 388 and $471 \mathrm{~nm}$, the shape of the E-PL 


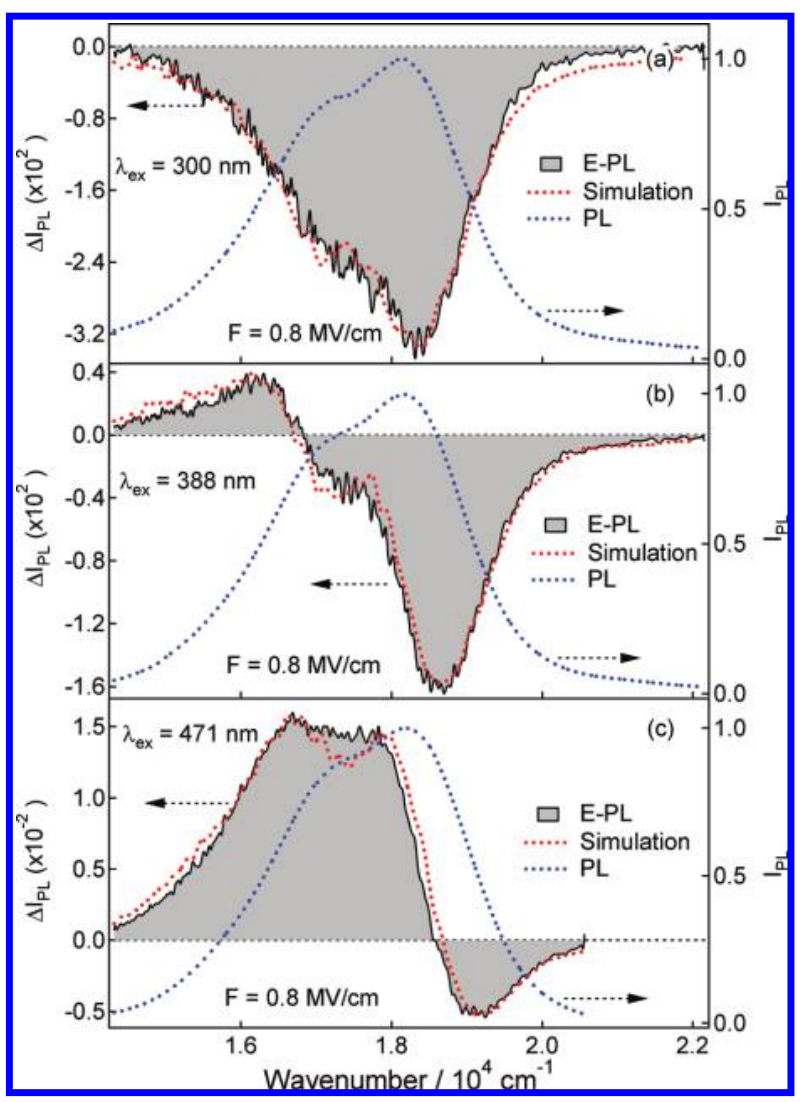

Figure 3. Electrophotoluminescence (shaded line) and photoluminescence spectra (dotted blue line) of an S3-PPV film obtained with excitation at (a) 300, (b) 388, and (c) $471 \mathrm{~nm}$ with a field strength of $0.8 \mathrm{MV} \mathrm{cm}^{-1}$ and the simulated spectra (dotted red line).

spectra does not match the PL spectrum. At $388 \mathrm{~nm}$, the E-PL spectrum is similar to the first derivative of the PL spectrum (green curves in Figure 2); that is, the E-PL spectrum is dominated by the Stark shift induced by a change of $\Delta \alpha$ following the emission. The magnitude of quenching at $388 \mathrm{~nm}$ was $0.69 \pm 0.25 \%$, much smaller than that observed on excitation at $300 \mathrm{~nm}$. With excitation at $471 \mathrm{~nm}$, the observed E-PL spectrum became mainly positive, indicating an enhancement rather than quenching of the PL intensity in the presence of electric fields. The magnitude of enhancement at $471 \mathrm{~nm}$ was $1.14 \pm 0.3 \%$ at a field strength of $0.8 \mathrm{MV} \mathrm{cm}^{-1}$.

The E-PL spectra observed with excitation at 300, 388, and $471 \mathrm{~nm}$ are reproduced by a linear combination of the zeroth and the first derivative of the PL spectrum based on eq 2 , as shown in Figure 3; the contribution of the second derivative of PL is negligible, indicating that $\Delta \alpha$ between the emitting and the ground states is dominant in the Stark shift of the E-PL spectra. According to eq $3, \Delta \bar{\alpha}$ is evaluated to be $840 \pm 10$ $\AA^{3} / f^{2}$, at any excitation wavelength employed. This value of $\Delta \bar{\alpha}$ is similar to that derived for PPV. ${ }^{13}$ As shown in Table 1 , the value of $\Delta \bar{\alpha}$ evaluated from the E-PL spectra of OPPV in a dilute solvent glass ranged from 500 to $3000 \AA^{3} / f^{2}$. The value of $\Delta \bar{\alpha}$ obtained from E-PL of MEH-PPV or OPPVs in a glassy solvent is similar to that obtained from E-A, indicating no difference of electronic properties between the absorbing and emitting states. ${ }^{12}$ In contrast, $\Delta \bar{\alpha}$ evaluated from the E-PL spectra of S3-PPV is about 1 order of magnitude larger than those determined from the E-A spectra, suggesting that the emitting state differs from the originally excited state that produces strong absorption near $22500 \mathrm{~cm}^{-1}$. The emitting state of S3-PPV seems to be similar to those of MEH-PPV or OPPVs in a glassy solvent, as far as the magnitude of $\Delta \bar{\alpha}$ is concerned.

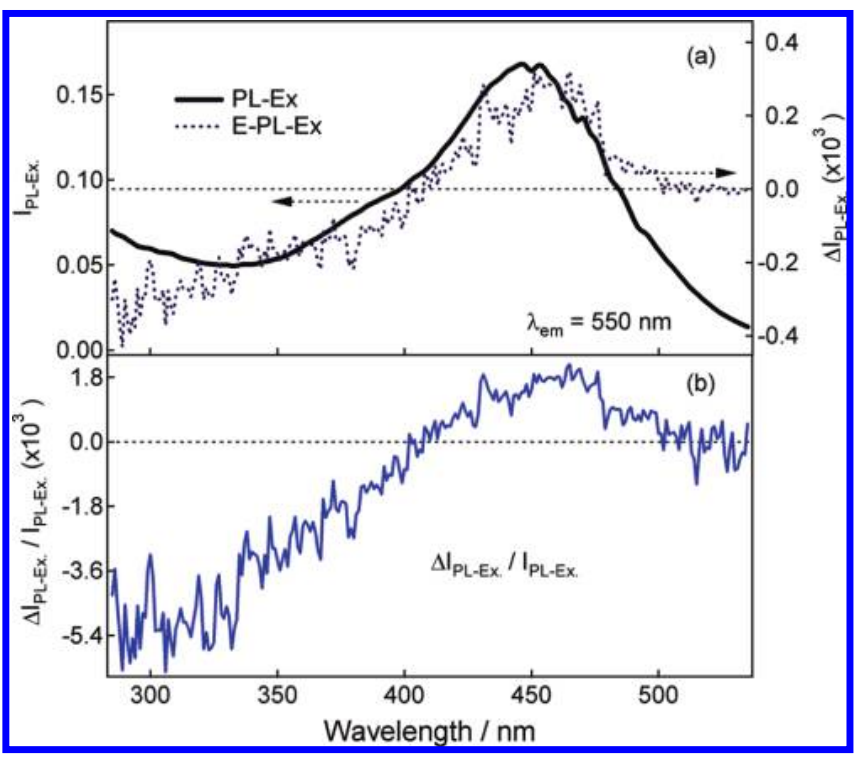

Figure 4. (a) Photoluminescence excitation spectrum (solid line) and electrophotoluminescence excitation spectrum (dotted line) of an S3PPV film observed at an emission wavelength of $550 \mathrm{~nm}$, and (b) the ratio of these two spectra. The applied field strength was $0.8 \mathrm{MV} \mathrm{cm}^{-1}$.

The zeroth derivative coefficient $\left(A_{\chi}^{\prime}\right)$ is -0.027 for excitation at $300 \mathrm{~nm},-0.007$ at $388 \mathrm{~nm}$, and +0.012 at $471 \mathrm{~nm}$ in the presence of a field strength of $0.8 \mathrm{MV} \mathrm{cm}^{-1}$ in the simulation. These magnitudes of quenching or enhancement are the same as those obtained on integrating the E-PL spectra at each excitation wavelength. These results clearly show that the fieldinduced change of PL intensity (PL quantum yield) depends on the excitation wavelength and that not only field-induced quenching but also field-induced enhancement occurs in the present $\pi$-conjugated polymer.

The dependence of the field-induced change of PL intensity on excitation wavelength was confirmed on measuring E-PL excitation spectra (E-PL-Ex) and PL excitation spectra (PLEx) of S3-PPV shown in Figure 4; these spectra were measured simultaneously, with an emission wavelength of $550 \mathrm{~nm}$. Note that E-PL-Ex spectra were measured as a function of excitation wavelength on monitoring the field-induced change in PL intensity at $550 \mathrm{~nm}$, at which the first derivative of the PL spectra is negligible. The ratio between E-PL-Ex and PL-Ex gives the field-induced change of quantum yield of PL at each excitation wavelength. The ratio of these two spectra, given in Figure $4 \mathrm{~b}$, clearly indicates that, as the excitation wavelength increases from $300 \mathrm{~nm}$, the field-induced PL quenching decreases monotonically. The quenching diminishes for excitation near $410 \mathrm{~nm}$, and field-induced enhancement was observed at excitation wavelengths in the range of $410-500 \mathrm{~nm}$. No change in PL intensity was observed at excitation wavelengths $>500 \mathrm{~nm}$, at which S3-PPV exhibits no absorption. The fieldinduced quenching for excitation at 300 and $388 \mathrm{~nm}$ and the field-induced enhancement for excitation at $471 \mathrm{~nm}$ shown in Figure 2 are consistent with the observed E-PL excitation spectra shown in Figure 4.

Free charges can quench the photoluminescence of conjugated polymers. ${ }^{21,22}$ As in the present samples, an insulator film of PMMA much thicker than the sample polymer film was inserted between the electrodes; it is unlikely that the observed quenching is caused by charge injection from the electrodes. The magnitude of the field-induced change of PL intensity of S3-PPV is proportional to the square of the applied field strength, indicating 


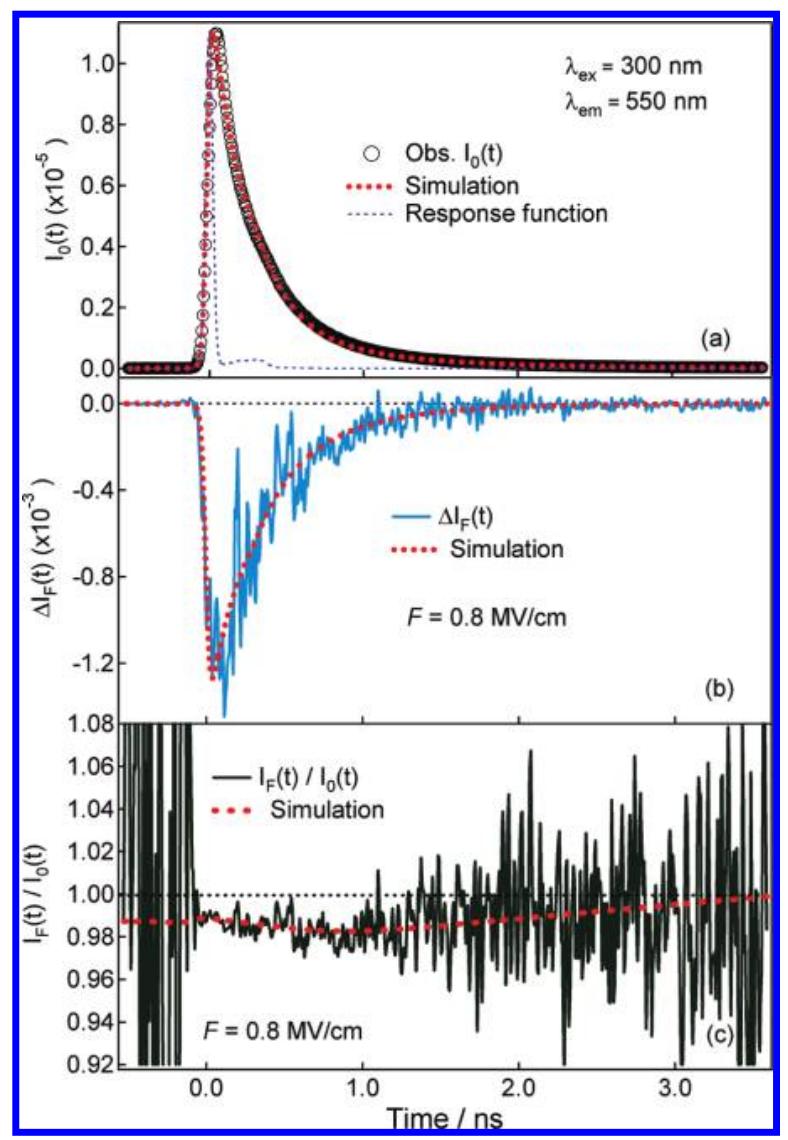

Figure 5. (a) Fluorescence decay (open circles) of an S3-PPV film observed at zero field, with the simulated curve (dotted red line) and the instrumental response function (dotted blue line). Excitation and emission wavelengths were 300 and $550 \mathrm{~nm}$, respectively. (b) Difference (solid line) between the fluorescence decays observed at $0.8 \mathrm{MV}$ $\mathrm{cm}^{-1}$ and at zero electric field, and the simulated difference (dotted line). (c) Ratio of the decay observed at $0.8 \mathrm{MV} \mathrm{cm}^{-1}$ relative to that at zero field (solid line), and the simulated one (dotted line).

that this field effect is independent of the injection efficiency of the hole or electron. As ITO and Al served as electrodes in the present experiments, this result indicates also that the observed PL quenching is unrelated to holes or electrons injected from the electrodes. ${ }^{23}$ Two mechanisms are possible for the field-induced changes of PL intensity: (i) an altered rate of nonradiative decay of the emitting state of S3-PPV, and (ii) an altered initial population of the emitting state following photoexcitation. The former condition leads to a decreased or increased PL lifetime, whereas the latter produces an altered population yield of the emitting state. Direct measurements of the field-induced change in temporally resolved profiles of PL/ fluorescence decay of S3-PPV films provide information to distinguish these two mechanisms. If the initial population of the emitting state of the S3-PPV fluorescence was unaffected by the presence of an electric field, the ratio of $I_{\mathrm{F}}(t) / I_{0}(t)$ between decay profiles in the absence $\left(I_{0}(t)\right)$ and presence of the field $\left(I_{\mathrm{F}}(t)\right)$ would be unity at $t=0$. Further, if the fluorescence lifetime was independent of the field, $I_{\mathrm{F}}(t) / I_{0}(t)$ would remain constant over the period.

Upon excitation at $300 \mathrm{~nm}$, the decays of PL of S3-PPV were monitored at $550 \mathrm{~nm}$ in the absence and presence of $F$ at 0.8 MV cm${ }^{-1}$; the difference, $\Delta I_{\mathrm{F}}(t)=I_{\mathrm{F}}(t)-I_{0}(t)$, and the ratio, $I_{\mathrm{F}}(t) / I_{0}(t)$, between these two decay profiles $I_{0}(t)$ and $I_{\mathrm{F}}(t)$ are shown in traces b and c of Figure 5. The contribution of the Stark shift is excluded from the observed field-induced change of decay profile on monitoring the PL at $550 \mathrm{~nm}$, as already

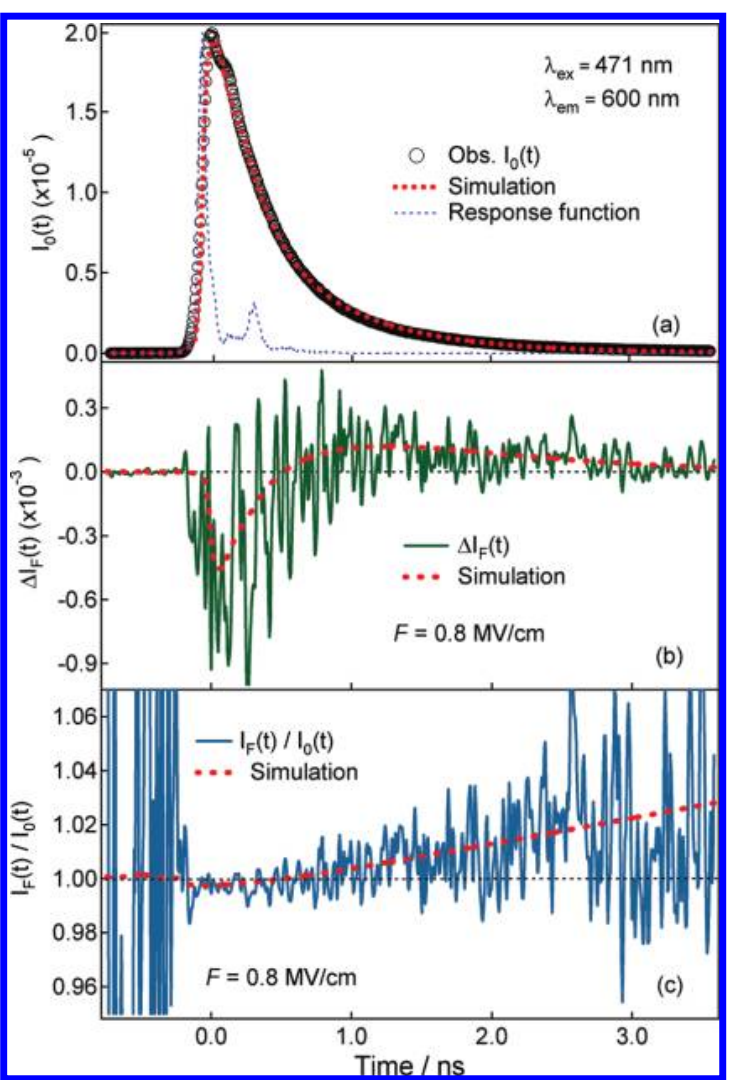

Figure 6. (a) Fluorescence decay (open circles) of an S3-PPV film observed at zero field, with the simulated curve (dotted red line) and the instrumental response function (dotted blue line). Excitation and emission wavelengths were 471 and $600 \mathrm{~nm}$, respectively. (b) Difference (solid line) between fluorescence decays observed at $0.8 \mathrm{MV} \mathrm{cm}^{-1}$ and zero electric field, and the simulated difference (dotted line). (c) Ratio of the decay observed at $0.8 \mathrm{MV} \mathrm{cm}^{-1}$ relative to that at zero field (solid line), and the simulated one (dotted line).

described in the measurements of the E-PL-Ex spectra, as the Stark shift is given by the first derivative of the PL spectrum, which gives zero value at $550 \mathrm{~nm}$. In Figure $5 \mathrm{~b}$, the difference between $I_{\mathrm{F}}(t)$ and $I_{0}(\mathrm{t})$, that is, $\Delta I_{\mathrm{F}}(t)$, and the integral of $\Delta I_{\mathrm{F}}(t)$ over the observation period are both negative, indicating that field-induced quenching of fluorescence occurs upon excitation at $300 \mathrm{~nm}$, in agreement with the observed steady-state E-PL spectrum. The ratio of $I_{\mathrm{F}}(t) / I_{0}(t)$ shown in Figure $5 \mathrm{c}$ is less than unity at $t=0$ following excitation at $300 \mathrm{~nm}$, indicating that the population of the emitting state is decreased due to the presence of $F$. Similar plots obtained on monitoring emission at $600 \mathrm{~nm}$ with excitation of S3-PPV at $471 \mathrm{~nm}$ are shown in Figure 6; $\Delta I_{\mathrm{F}}(t)$ first becomes slightly negative but increases to become positive after $0.8 \mathrm{~ns}$ (Figure $6 \mathrm{~b}$ ). The integrated value of $\Delta I_{\mathrm{F}}(t)$ is positive, indicating a field-induced enhancement of fluorescence upon excitation at $471 \mathrm{~nm}$, consistent with the observed steady-state E-PL spectrum. The fact that the ratio of $I_{\mathrm{F}}(t) / I_{0}(t)$ shown in Figure $6 \mathrm{c}$ is roughly unity at $t=0$ implies that the initial population of the emitting state is nearly unaffected by $F$. At a later period, $I_{\mathrm{F}}(t) / I_{0}(t)$ increases with time, indicating an increase in fluorescence lifetime in the presence of $F$. A detailed analysis of the profiles of fluorescence decay thus provides information on the variation of population and lifetime of PL of S3-PPV upon excitation at various wavelengths in the presence of $F$.

The decay profiles of PL in Figures 5a and 6a were simulated assuming a biexponential decay, $A_{1} \exp \left(-t / \tau_{1}\right)+A_{2} \exp \left(-t / \tau_{2}\right)$, in which $\tau_{1}$ and $\tau_{2}$ are the lifetimes and $A_{1}$ and $A_{2}$ are the pre- 
TABLE 2: Fluorescence Lifetimes and Pre-exponential Factors (parentheses) of S3-PPV at Zero Field and at 0.8 $M V \mathrm{~cm}^{-1}$ for $300 \mathrm{~nm}$ and $471 \mathrm{~nm}$ Excitations

\begin{tabular}{cccc}
\hline $\begin{array}{c}\lambda_{\mathrm{ex}} \\
(\mathrm{nm})\end{array}$ & $\begin{array}{c}F \\
\left(\mathrm{MV} \mathrm{cm}^{-1}\right)\end{array}$ & $\tau_{1}(\mathrm{ps})$ & $\tau_{2}(\mathrm{ps})$ \\
\hline 300 & 0 & $215 \pm 15(0.879 \pm 0.002)$ & $751 \pm 40(0.121 \pm 0.001)$ \\
& 0.8 & $215 \pm 15(0.870 \pm 0.002)$ & $755 \pm 30(0.118 \pm 0.001)$ \\
471 & 0 & $224 \pm 15(0.642 \pm 0.002)$ & $719 \pm 25(0.357 \pm 0.001)$ \\
& 0.8 & $224 \pm 15(0.642 \pm 0.002)$ & $724 \pm 25(0.356 \pm 0.002)$
\end{tabular}

exponential factors. The results for the decays observed at zero field and at $0.8 \mathrm{MV} \mathrm{cm}{ }^{-1}$ with excitation at 300 and $471 \mathrm{~nm}$ are shown in Table 2. The fluorescence lifetimes observed with excitation at $300 \mathrm{~nm}$ (i.e., $\tau_{1}=215 \pm 15 \mathrm{ps}$ and $\tau_{2}=751 \pm 40$ ps) are similar to those reported for MEH-PPV at zero field (i.e., $\tau_{1}=212 \mathrm{ps}$ and $\tau_{2}=763 \mathrm{ps}$ ). ${ }^{11}$ On excitation at $300 \mathrm{~nm}$, the pre-exponential factor $A_{1}$ of the rapid component decreases from $0.879 \pm 0.002$ to $0.870 \pm 0.002$, and the lifetime of the slowly decaying component increases slightly in the presence of $F$. In contrast, upon excitation at $471 \mathrm{~nm}$, the pre-exponential factors are nearly unaffected, but the lifetime of the slower component $\tau_{2}$ similarly increases slightly on application of $F$. These results are illustrated in the plots of $I_{\mathrm{F}}(t) / I_{0}(t)$ in Figures $5 \mathrm{c}$ and $6 \mathrm{c}$. The dominant mode of electric field-assisted PL quenching in S3-PPV upon excitation at $300 \mathrm{~nm}$ is thus a decreased population, whereas the dominant mode of the fieldassisted PL enhancement upon excitation at $471 \mathrm{~nm}$ is an increased lifetime. It should be stressed that a slight increase of the slow fluorescence lifetime occurs by application of electric fields even with excitation at $300 \mathrm{~nm}$ (see Table 2). As shown in Figure 5, however, the intensity enhancement induced by lifetime lengthening is drowned in the overall intensity decrease caused by the field-induced depopulation of the emitting state.

As described above, electric-field-induced enhancement or quenching of PL of S3-PPV has been observed, depending on the excitation wavelength. To the best of our knowledge, this is the first case of such a field-induced enhancement of PL reported for a $\pi$-conjugated polymer. As discussed above, the field-induced enhancement of PL observed on excitations at 471 $\mathrm{nm}$ results from the field-induced decrease of the nonradiative decay rate at the emitting state. At these excitation wavelengths, the relaxation from the photoexcited state to the emitting state is likely dominant. From the emitting state, a nonradiative process besides the fluorescence process may exist. As the nonradiative decay process from the emitting state, intersystem crossing to nearby triplet states can be considered. If the molecular polarizability as well as the dipole moment at the emitting state (singlet state) is very different from the ones of the triplet states, the energy separation between the emitting state and nearby triplet states is largely shifted by application of electric field. As a result, the intersystem crossing may be decelerated by the applied electric field, resulting in the increase of the fluorescence intensity. In contrast with the enhancement, electric-field-induced quenching of PL was reported in conjugated polymers, such as in poly( $p$-terphenylene vinylene $),{ }^{24}$ MEH-PPV,${ }^{11}$ poly( $p$-phenylphenylenevinylene) blended with polycarbonate, ${ }^{25-27}$ or poly(p-phenylenevinylene).$^{28}$ As the origin of the field-induced quenching of PL reported so far, an exciton model and a band model have been proposed. A breaking of excitons into electron-hole pairs in the presence of electric fields, which results in photocarrier generation in a secondary process, is suggested in the former model, whereas direct photogeneration of free charge carriers via an interband transition is considered in the latter model. In the present experiments, the magnitude of the field-induced quenching of
PL, which might be correlated with photocarrier generation, shows no maximum near the absorption edge and increases monotonically with increasing excitation energy. The fieldinduced quenching of PL of S3-PPV seems hence to be interpretable in terms of an exciton model; a field-assisted generation of electron-hole pairs occurs from the exciton state (a nonrelaxed excited state) of S3-PPV, resulting in the quenching of PL. The field-induced dissociation of an exciton with a lifetime of $\sim 1$ ps was directly observed for a blended system of poly(phenylphenylenevinylene) and polycarbonate with a field strength of $\sim 2 \mathrm{MV} \mathrm{cm}{ }^{-1} .27$

In the present measurements of time-resolved emission, only the decrease of the pre-exponential factor, that is, the fieldinduced decrease of the population of the emitting state, was detected in connection with field-induced quenching, probably because the present time resolution ( $\sim 20 \mathrm{ps})$ was inadequate to detect the change in rise, from which the dissociation rate can be estimated. With increasing excitation energy, the magnitude of quenching of PL increases, indicating that both the rate and the efficiency of the field-assisted dissociation into electron-hole pairs from excitons increases with increasing excitation energy. With increasing excitation energy, electronhole pairs can acquire larger excess energy, resulting in a large distance between electron and hole in thermal equilibrium. ${ }^{29}$ Field-assisted dissociation becomes increasingly efficient with increasing excitation energy, in agreement with the present experiments. A similar dependence on excitation energy, that is, an efficient field-induced quenching PL on excitation into states at higher energies, was observed in polyfluorene films. ${ }^{7}$ For MEH-PPV, application of an electric field decreases the fluorescence lifetime, indicating that the dominant mode of fluorescence quenching is lifetime quenching. ${ }^{11}$ In contrast, the electric field effect on the lifetime of PL of S3-PPV is very small, but the quenching of PL occurs with excitation into higher excited states, suggesting that only the nonradiative process at the photoexcited state that competes with relaxation to the emitting state is well affected by electric fields. The nonradiative process of S3-PPV under consideration is assigned to a fieldassisted generation of electron-hole pairs, as described above. On the basis of the dependences on concentration and chain length, the field-induced quenching of emission intensity occurred also as a result of the field-induced change in intrachain dynamics; an energy shift of the relaxed excited state caused by the electric field results in an enhanced nonradiative relaxation such as internal conversion, leading to the fieldinduced quenching of fluorescence, ${ }^{13}$ which differs markedly from the present field effects on the dynamics of the emitting state.

C. Photoirradiation Effect on PL of an S3-PPV Film in Ambient Air. Figure 7a shows PL spectra of an S3-PPV film recorded continuously under photoexcitation at $350 \mathrm{~nm}(4 \mathrm{~mW}$ $\mathrm{cm}^{-2}$ ) under ambient air; the direction of the scanning is increasing wavelength. By photoirradiation, the intensity of the PL spectra rapidly decreased, and the wavelength of the maximal intensity, which was initially $551 \mathrm{~nm}$, shifted toward a shorter wavelength. Figure $7 \mathrm{~b}$ shows PL excitation spectra of an S3PPV film under ambient air that were recorded continuously by monitoring the emission at $550 \mathrm{~nm}$; the direction of the scanning is increasing wavelength. Throughout the photoirradiation, the discrete features of the excitation spectra remained unchanged except for a rapidly decreased intensity, whereas the broad continuum showed a rapid decrease in intensity and its maximum shifted toward a shorter wavelength. 


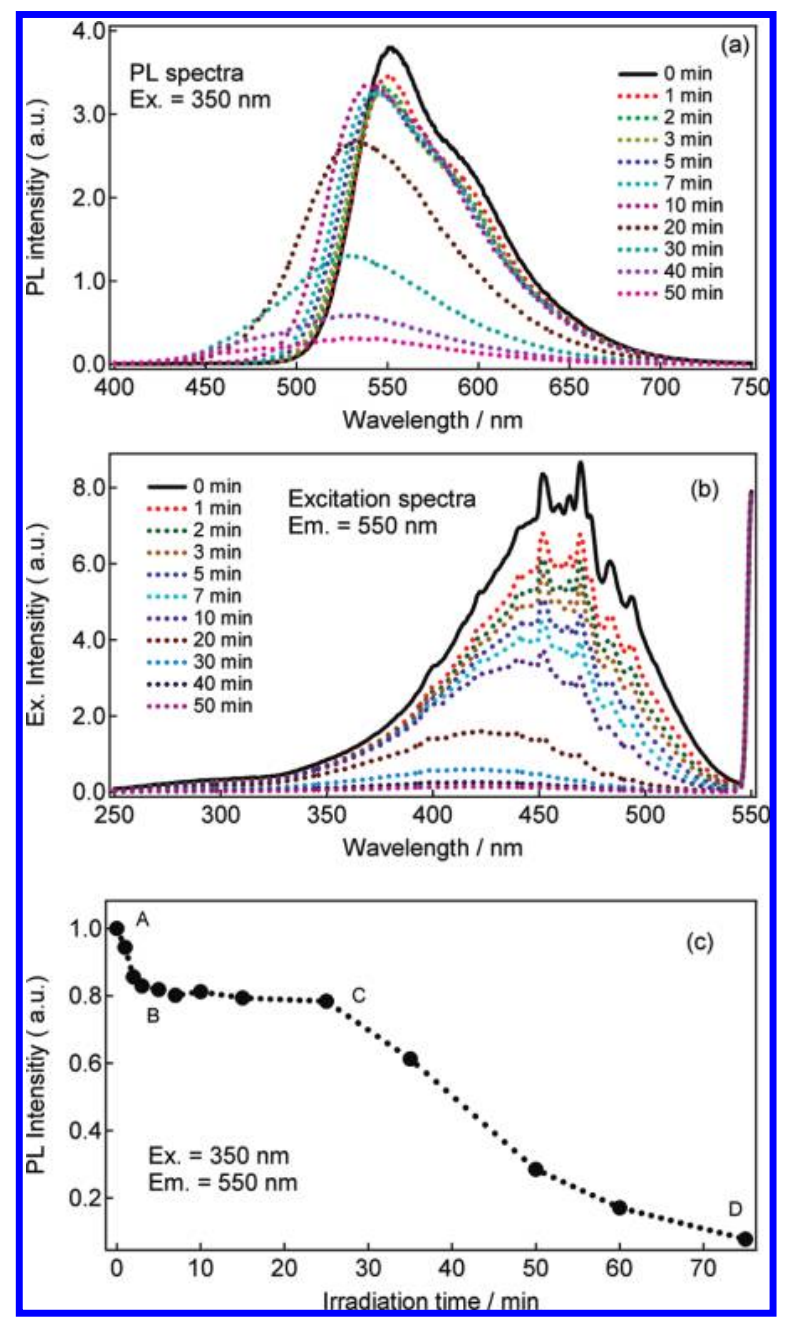

Figure 7. (a) Photoluminescence (PL) spectra and (b) PL excitation spectra of an S3-PPV film for various periods of irradiation near 298 $\mathrm{K}$ under atmospheric conditions. (c) PL intensity of an S3-PPV film plotted as a function of duration of irradiation, with an excitation wavelength of $350 \mathrm{~nm}$. No correction was made for the PL excitation spectra for the intensity distribution of the excitation light as a function of wavelength.

Plots of the PL intensity of the S3-PPV film at $550 \mathrm{~nm}$ were obtained as a function of duration of photoirradiation with excitation at $350 \mathrm{~nm}$. The results are shown in Figure 7c. The photoirradiation effect on PL spectra comprises three stages. In the first stage (i.e., $\mathrm{A}-\mathrm{B}$ in Figure $7 \mathrm{c}$ ), the PL intensity greatly decreases, perhaps because of a creation of defects in the main chain by atmospheric oxygen. In the second stage (i.e., B-C in Figure 7c), the PL intensity remains constant, but the maximum intensity of the fluorescence spectrum shifts to a shorter wavelength. A decrease of the conjugation length due to scission of the main chain upon photo-oxidation and the exciton confinement might explain the observed behavior. In the third stage (i.e., C-D in Figure 7c), the PL intensity further decreases, which may be ascribed to the chemical defects created in the remaining chain of the S3-PPV polymer. Under ambient air, oxygen may damage the main chain through chemical reactions of S3-PPV in the excited state and decrease the chain length.

\section{Summary}

The effects of external electric field and irradiation on absorption and photoluminescence spectra of S3-PPV films were investigated. Both E-A and E-PL spectra show quadratic Stark shifts, which demonstrate a large difference in molecular polarizability between the emitting and ground states. The E-PL response of S3-PPV under vacuum depends on the excitation wavelength. At $300 \mathrm{~nm}$, the PL of S3-PPV is quenched in the presence of $F$ through a diminished population of the emitting states, perhaps because of enhanced dissociation before nonradiative relaxation to the emitting states. Application of the electric field dissociates excitons into electron-hole pairs that become charge carriers. ${ }^{11}$ The field-induced quenching of PL increases with increasing wavenumber of excitation light, indicating that the efficiency of the field-assisted dissociation increases with increasing excitation energy. These results clearly show that the field-induced generation of electron-hole pairs through excitons of the conjugated polymer occurs from nonrelaxed higher vibrational levels of the excited state in competition with relaxation to the emitting state. With increasingly excess vibrational energy, the efficiency of generation of field-induced electron-hole pairs increases. On excitation at 471 $\mathrm{nm}$, at which the PL intensity was enhanced with electric fields, the lifetime of PL increased on application of electric fields. These results show that the nonradiative decay rate at the emitting state of PL is diminished by an electric field, which results in an increased lifetime and enhanced PL quantum yield (intensity) induced by the field. Irradiation of S3-PPV under ambient air caused a rapid degradation of the polymer film that shows much weaker emission with a maximum shifted toward shorter wavelength.

Acknowledgment. M.S.M. thanks Japan Society for the Promotion of Science (JSPS) for a JSPS-Postdoctoral fellowship and for a Grant-in-Aid for JSPS fellows. This work was also supported by the Interchange Association, Japan (Japan-Taiwan Joint Research Program).

Supporting Information Available: Additional experimental details. This material is available free of charge via the Internet at http://pubs.acs.org.

\section{References and Notes}

(1) Jung, S. J.; Cho, Y. R.; Jin, S. H.; Kim, S. C.; Shin, W. S.; Lee, J. W.; Gal, Y. S. Curr. Appl. Phvs. 2007, 7, 375.

(2) Burroughes, J. H.; Bradley, D. D. C.; Brown, A. R.; Marks, R. N.; Mackay, K.; Friend, R. H.; Burns, P. L.; Holmes, A. B. Nature 1990, 347, 539.

(3) Bernius, M. T.; Inbasekaran, M.; O’Brien, J.; Wu, W. Adv. Mater. 2000, 12, 1737.

(4) Zyung, T.; Kim, J.-J. Appl. Phvs. Lett. 1995, 67, 3420.

(5) Tada, K.; Onoda, M. J. Appl. Phvs. 1999, 86, 3134.

(6) Khan, M. I.; Renak, M. L.; Bazan, G. C.; Popovic, Z. J. Am. Chem. Soc. 1997, 119, 5344.

(7) Mehata, M. S.; Hsu, C. S.; Lee, Y. P.; Ohta, N. J. Phvs. Chem. C 2009, 113, 11907.

(8) Chen, K. B.; Chen, M. H.; Yang, S. H.; Hsieh, C. H.; Hsu, C. S.; Chen, C. C.; Chien, H. J. J. Polym. Sci., Part A: Polym. Chem. 2006, 44, 5378.

(9) Chen, K. B.; Li, H. C.; Chen, C. K.; Yang, S. H.; Hsieh, B. R.; Hsu, C. S. Macromolecules 2005, 38, 8617.

(10) Martin, S. J.; Mellor, H.; Bradley, D. D. C.; Burn, P. L. Opt. Mater. 1998, $9,88$.

(11) Khan, M. I.; Bazan, G. C.; Popovic, Z. D. Chem. Phys. Lett. 1998, $298,309$.

(12) Smith, T. M.; Hazelton, N.; Peteanu, L. A.; Wildeman, J. J. Phys. Chem. B 2006, 110, 7732 .

(13) Smith, T. M.; Kim, J.; Peteanu, L. A.; Wildeman, J. J. Phys. Chem. C 2007, 111, 10119 .

(14) Umeuchi, S.; Nishimura, Y.; Yamazaki, I.; Murakami, H.; Yamashita, M.; Ohta, N. Thin Solid Films 1997, 311, 239. 479. 
(16) Liptay, W. In Excited States; Lim, E. C., Ed.; Academic Press: New York, 1974; Vol. 1, p 129.

(17) Bublit, G. U.; Boxer, S. G. Annu. Rev. Phys. Chem. 1997, 48, 213.

(18) Jalviste, E.; Ohta, N. J. Photochem. Photobiol. C 2007, 8, 30.

(19) Martin, S. J.; Bradley, D. D. C.; Lane, P. A.; Mellor, H. Phys. Rev. B 1999, 59, 15133.

(20) Traiphol, R.; Charoenthai, N.; T. Srikhirin, T.; Kerdcharoen, T.; Osotchan, T.; Maturos, T. Polvmer 2007, 48, 813.

(21) Deussen, N.; Scheidler, M.; Bässler, H. Synth. Met. 1995, 73, 123.

(22) Scheblykin, I.; Zoriniants, G.; Hofkens, J.; De Feyter, S.; Van der Auweraer, M.; De Schryver, F. C. ChemPhysChem. 2003, 4, 260.

(23) Park, S.-J.; Gesquiere, A. J.; Yu, J.; Barbara, P. F. J. Am. Chem. Soc. 2004, 126, 4116.
(24) Pfeffer, N.; Neher, D.; Remmers, M.; Poga, C.; Hopmeier, M.; Mahrt, R. Chem. Phvs. 1998, 227, 167.

(25) Arkhipov, V. I.; Bässler, H.; Deussen, M.; Göbel, E. O.; Kersting,

R.; Kurz, H.; Lemmer, U.; Mahrt, R. F. Phvs. Rev. B 1995, 52, 4932.

(26) Deussen, M.; Scheidler, M.; Bässler, H. Synth. Met. 1995, 73, 123.

(27) Kersting, R.; Lemmer, U.; Deussen, M.; Bakker, H. J.; Mahrt, R. F.; Kurz, H.; Arkhipov, V. I.; Bässler, H.; Göbel, E. O. Phys. Rev. Lett. 1994, 73,1440 .

(28) Lee, C. H.; Yu, G.; Heeger, A. J. Phvs. Rev. 1993, 47, 15543.

(29) Onsager, L. J. Chem. Phvs. 1934, 2, 599; Phys. Rev. 1938, 54, 39.

JP912199P 